\title{
BLOW-UP OF SOLUTIONS OF A SEMILINEAR HEAT EQUATION WITH A MEMORY TERM
}

\author{
SALIM A. MESSAOUDI
}

Received 26 May 2004

We consider an initial boundary value problem related to the equation $u_{t}-\Delta u+\int_{0}^{t} g(t-$ $s) \Delta u(x, s) d s=|u|^{p-2} u$ and prove, under suitable conditions on $g$ and $p$, a blow-up result for certain solutions with positive initial energy.

\section{Introduction}

In this paper, we are concerned with the finite-time blow-up of solutions for the initial boundary value problem

$$
\begin{gathered}
u_{t}-\Delta u+\int_{0}^{t} g(t-s) \Delta u(x, s) d s=|u|^{p-2} u, \quad x \in \Omega, t>0, \\
u(x, t)=0, \quad x \in \partial \Omega, t \geq 0 \\
u(x, 0)=u_{0}(x), \quad x \in \Omega
\end{gathered}
$$

where $g: \mathbb{R}_{+} \rightarrow \mathbb{R}_{+}$is a bounded $C^{1}$ function, $p>2$, and $\Omega$ is a bounded domain of $\mathbb{R}^{n}$ $(n \geq 1)$, with a smooth boundary $\partial \Omega$.

This equation arises from a variety of mathematical models in engineering and physical sciences. For example, in the study of heat conduction in materials with memory, the classical Fourier's law of heat flux is replaced by the following form:

$$
q=-d \nabla u-\int_{-\infty}^{t} \nabla[k(x, t) u(x, \tau)] d \tau
$$

where $u$ is the temperature, $d$ is the diffusion coefficient, and the integral term represents the memory effect in the material. The study of this type of equations has drawn a considerable attention, see $[3,4,10,12,13]$. From a mathematical point of view, one would expect the integral term to be dominated by the leading term in the equation. Therefore, the theory of parabolic equations applies to this type of equations.

In the absence of the memory term $(g=0)$, problem $(1.1)$ has been studied by various authors and several results concerning global and nonglobal existence have been established. For instance, in the early 1970s, Levine [6] introduced the concavity method and 
showed that solutions with negative energy blow up in finite time. Later, this method was improved by Kalantarov and Ladyzhenskaya [5] to accommodate more general situations. Ball [2] also studied (1.1) with $f(u, \nabla u)$ instead of $|u|^{p-2} u$ and established a nonglobal existence result in bounded domains. This result had been extended to unbounded domains by Alfonsi and Weissler [1].

For the quasilinear case, Junning in [14] studied

$$
\begin{gathered}
u_{t}-\operatorname{div}\left(|\nabla u|^{m-2} \nabla u\right)=f(u), \quad x \in \Omega, t>0, \\
u(x, t)=0, \quad x \in \partial \Omega, t \geq 0, \\
u(x, 0)=u_{0}(x), \quad x \in \Omega,
\end{gathered}
$$

and established a global existence result. He also proved a nonglobal existence result under the condition

$$
\frac{1}{m} \int_{\Omega}\left|\nabla u_{0}(x)\right|^{m} d x-\int_{\Omega} F\left(u_{0}(x)\right) d x \leq-\frac{4(m-1)}{m T(m-2)^{2}} \int_{\Omega} u_{0}^{2}(x) d x
$$

where $F(u)=\int_{0}^{u} f(s) d s$. More precisely he showed that if there exists $T>0$, for which (1.2) holds, then the solution blows up in a time less than $T$. This type of results have been extensively generalized and improved by Levine, Park, and Serrin in [7], where the authors proved some global, as well as nonglobal, existence theorems. Their result, when applied to problem (1.3), requires that

$$
\frac{1}{m} \int_{\Omega}\left|\nabla u_{0}(x)\right|^{m} d x-\int_{\Omega} F\left(u_{0}(x)\right) d x<0 .
$$

We note that the inequality (1.5) implies (1.4). In a note, Messaoudi [8] extended the blow-up result to a solution with an initial datum satisfying

$$
\frac{1}{m} \int_{\Omega}\left|\nabla u_{0}(x)\right|^{m} d x-\int_{\Omega} F\left(u_{0}(x)\right) d x \leq 0
$$

In the present work, we consider (1.1) and show that, for suitable conditions on $p$ and $g$, the blow-up can be obtained even for some solutions with positive initial energy. The present paper improves the one in [8] as it is only a special case.

\section{Blow-up}

In order to state and prove our result, we introduce the "modified" energy functional

$$
E(t)=\frac{1}{2}(g \diamond \nabla u)(t)+\frac{1}{2}\left(1-\int_{0}^{t} g(s) d s\right)\|\nabla u(t)\|_{2}^{2}-\frac{1}{p}\|u(t)\|_{p}^{p},
$$

where

$$
(g \diamond v)(t)=\int_{0}^{t} g(t-\tau)\|v(t)-v(\tau)\|_{2}^{2} d \tau
$$


For the relaxation function $g$ and the number $p$, we assume that

$$
\begin{gathered}
g(s) \geq 0, \quad g^{\prime}(s) \leq 0, \quad 1-\int_{0}^{\infty} g(s) d s=l>0, \\
2<p \leq \frac{2(n-1)}{n-2}, \quad n>2, p>2, n=1,2 .
\end{gathered}
$$

We also set

$$
\alpha=B^{-p /(p-2)}, \quad E_{1}=\left(\frac{1}{2}-\frac{1}{p}\right) \alpha^{2},
$$

where $B=C_{*} / l$ for $C_{*}$ the best constant of the Sobolev embedding $H_{0}^{1}(\Omega) \hookrightarrow L^{p}(\Omega)$.

By multiplying the equation in (1.1) by $u_{t}$ and integrating over $\Omega$, we get, after some manipulations, see [9],

$$
\frac{d}{d t} E(t)=-\left(\frac{1}{2} g(t)\|\nabla u(t)\|_{2}^{2}-\frac{1}{2}\left(g^{\prime} \diamond \nabla u\right)(t)+\int_{\Omega}\left|u_{t}\right|^{2} u_{t} d x\right) \leq 0
$$

for regular solutions. The same result can be established, for almost every $t$, by a simple density argument.

Similar to [11], we give a definition for a strong solution of (1.1).

Definition 1. A strong solution of $(1.1)$ is a function $u \in C\left([0, T) ; H_{0}^{1}(\Omega)\right) \cap C^{1}([0, T)$; $L^{2}(\Omega)$ ), satisfying (2.6) and

$$
\int_{0}^{t} \int_{\Omega}\left(\nabla u \cdot \nabla \phi-\int_{0}^{s} \nabla u(\tau) \cdot \nabla \phi(s) d \tau+u_{t} \phi-|u|^{p-2} u \phi\right) d x d s=0
$$

for all $t$ in $[0, T)$ and all $\phi$ in $C\left([0, T), H_{0}^{1}(\Omega)\right)$.

Remark 2.1. Condition (2.4) is needed so that $|u|^{p-2} u \in L^{2}(\Omega)$; hence $\int_{\Omega}|u|^{p-2} u \phi d x$ makes sense. The condition $1-\int_{0}^{\infty} g(s) d s=l>0$ is necessary to guarantee the parabolicity of system (1.1).

LEMma 2.2. Let $u$ be a strong solution of (1.1) with initial data satisfying

$$
E(0)<E_{1}, \quad\left\|\nabla u_{0}\right\|_{2}>\alpha
$$

Then there exists a constant $\beta>\alpha$ such that

$$
\begin{gathered}
{\left[\left(1-\int_{0}^{t} g(s) d s\right)\|\nabla u\|_{2}^{2}+(g \circ \nabla u)(t)\right]^{1 / 2} \geq \beta} \\
\|u\|_{p} \geq B \beta \quad \forall t \in[0, T) .
\end{gathered}
$$


90

Blow-up of solutions of a heat equation

Proof. We first note that, by (2.1) and the Sobolev embedding, we have

$$
\begin{aligned}
E(t)= & \frac{1}{2}\left(1-\int_{0}^{t} g(s) d s\right)\|\nabla u\|_{2}^{2}+\frac{1}{2}(g \circ \nabla u)(t)-\frac{1}{p}\|u\|_{p}^{p} \\
\geq & \frac{1}{2}\left(1-\int_{0}^{t} g(s) d s\right)\|\nabla u\|_{2}^{2}+\frac{1}{2}(g \circ \nabla u)(t)-\frac{1}{p} B^{p} l^{p}\|\nabla u\|_{2}^{p} \\
\geq & \frac{1}{2}\left(1-\int_{0}^{t} g(s) d s\right)\|\nabla u\|_{2}^{2}+\frac{1}{2}(g \circ \nabla u)(t) \\
& -\frac{B^{p}}{p}\left[\left(1-\int_{0}^{t} g(s) d s\right)\|\nabla u\|_{2}^{2}+(g \circ \nabla u)(t)\right]^{p / 2} \\
= & \frac{1}{2} \zeta^{2}-\frac{B^{p}}{p} \zeta^{p}=h(\zeta),
\end{aligned}
$$

where

$$
\zeta=\left[\left(1-\int_{0}^{t} g(s) d s\right)\|\nabla u\|_{2}^{2}+(g \circ \nabla u)(t)\right]^{1 / 2}
$$

It is easy to verify that $h$ is increasing for $0<\zeta<\alpha$, decreasing for $\zeta>\alpha, h(\zeta) \rightarrow-\infty$ as $\zeta \rightarrow+\infty$, and

$$
h(\alpha)=\left(\frac{1}{2}-\frac{1}{p}\right) B^{-2 p /(p-2)}=E_{1}
$$

where $\alpha$ is given in (2.8). Therefore, since $E(0)<E_{1}$, there exists $\beta>\alpha$ such that $h(\beta)=$ $E(0)$.

By using (2.11) we have

$$
h\left(\left\|\nabla u_{0}\right\|_{2}\right) \leq E(0)=g(\beta),
$$

which implies that $\left\|\nabla u_{0}\right\|_{2} \geq \beta$.

Now to establish (2.9), we suppose by contradiction that

$$
\left[\left(1-\int_{0}^{t_{0}} g(s) d s\right)\|\nabla u\|_{2}^{2}+(g \circ \nabla u)\left(t_{0}\right)\right]^{1 / 2}<\beta,
$$

for some $t_{0}>0$ and, by the continuity of

$$
\left(1-\int_{0}^{t} g(s) d s\right)\|\nabla u\|_{2}^{2}+(g \circ \nabla u)(t)
$$

we can choose $t_{0}$ such that

$$
\left[\left(1-\int_{0}^{t_{0}} g(s) d s\right)\|\nabla u\|_{2}^{2}+(g \circ \nabla u)\left(t_{0}\right)\right]^{1 / 2}>\alpha .
$$


Again the use of (2.11) leads to

$$
E\left(t_{0}\right) \geq h\left(\left[\left(1-\int_{0}^{t_{0}} g(s) d s\right)\|\nabla u\|_{2}^{2}+(g \circ \nabla u)\left(t_{0}\right)\right]^{1 / 2}\right)>h(\beta)=E(0) .
$$

This is impossible since $E(t) \leq E(0)$, for all $t \in[0, T)$. Hence (2.9) is established.

To prove (2.10), we exploit (2.1) and (2.6) to obtain

$$
\frac{1}{2}\left[\left(1-\int_{0}^{t} g(s) d s\right)\|\nabla u\|_{2}^{2}+(g \circ \nabla u)(t)\right] \leq E(0)+\frac{1}{p}\|u\|_{p}^{p} .
$$

Consequently

$$
\begin{aligned}
\frac{1}{p}\|u\|_{p}^{p} & \geq \frac{1}{2}\left[\left(1-\int_{0}^{t} g(s) d s\right)\|\nabla u\|_{2}^{2}+(g \circ \nabla u)(t)\right]-E(0) \\
& \geq \frac{1}{2} \beta^{2}-E(0) \\
& \geq \frac{1}{2} \beta^{2}-h(\beta)=\frac{B^{p}}{p} \beta^{p} .
\end{aligned}
$$

Therefore (2.20) yields the desired result. The proof is completed.

Theorem 2.3. Assume that (2.3) and (2.4) hold. Given $u_{0} \in H_{0}^{1}(\Omega)$ satisfying

$$
\left\|\nabla u_{0}\right\|_{2}>\alpha, \quad E(0)<E_{1}
$$

if

$$
\int_{0}^{\infty} g(s) d s<\frac{1-c_{0}}{1-(3 / 4) c_{0}}, \quad c_{0}=\frac{2+(p-2)(\alpha / \beta)^{p}}{p}<1
$$

then any strong solution of (1.1) blows up in finite time.

Proof. We define

$$
L(t)=\frac{1}{2} \int_{\Omega} u^{2}(x, t) d x
$$

and differentiate $L$ to get

$$
\begin{aligned}
L^{\prime}(t)= & \int_{\Omega} u u_{t}(x, t) d x \\
= & \int_{\Omega} u \Delta u d x-\int_{\Omega} u(x, t) \int_{0}^{t} g(t-s) \Delta u(x, s) d s d x+\int_{\Omega}|u|^{p} d x \\
= & -\int_{\Omega}|\nabla u|^{2} d x+\int_{\Omega} \int_{0}^{t} g(t-s) \nabla u(x, t) \cdot \nabla u(x, s) d s d x+\int_{\Omega}|u|^{p} d x \\
\geq & -\int_{\Omega}|\nabla u|^{2} d x+\int_{0}^{t} g(t-s)|| \nabla u(t)||_{2}^{2} d \tau+\int_{\Omega}|u|^{p} d x \\
& -\int_{0}^{t} g(t-s) \int_{\Omega}|\nabla u(t) \cdot[\nabla u(s)-\nabla u(t)]| d x d \tau .
\end{aligned}
$$


By using Schwarz inequality, (2.24) takes the form

$$
\begin{aligned}
L^{\prime}(t) \geq & \int_{\Omega}|u|^{p} d x-\left(1-\int_{0}^{t} g(s) d s\right)\|\nabla u(t)\|_{2}^{2} \\
& -\int_{0}^{t} g(t-\tau)\|\nabla u(t)\|{ }_{2}\|\nabla u(\tau)-\nabla u(t)\|_{2} d \tau .
\end{aligned}
$$

By applying Young's inequality to the last term of (2.25), we arrive at

$$
L^{\prime}(t) \geq \int_{\Omega}|u|^{p} d x-\left[1-\frac{3}{4} \int_{0}^{t} g(s) d s\right]\|\nabla u(t)\|_{2}^{2}-(g \diamond \nabla u)(t) .
$$

We then substitute for $\|\nabla u(t)\|_{2}^{2}$ from (2.1); hence (2.26) becomes

$$
\begin{aligned}
L^{\prime}(t) \geq & \int_{\Omega}|u|^{p} d x+2 \frac{\left[1-(3 / 4) \int_{0}^{t} g(s) d s\right]}{\left(1-\int_{0}^{t} g(s) d s\right)} H(t)-2 \frac{\left[1-(3 / 4) \int_{0}^{t} g(s) d s\right]}{\left(1-\int_{0}^{t} g(s) d s\right)} E_{1} \\
& +\left(\frac{1-(3 / 4) \int_{0}^{t} g(s) d s}{\left(1-\int_{0}^{t} g(s) d s\right)}-1\right)(g \diamond \nabla u)(t) \\
& -\frac{2}{p} \frac{1-(3 / 4) \int_{0}^{t} g(s) d s}{\left(1-\int_{0}^{t} g(s) d s\right)} \int_{\Omega}|u|^{p} d x .
\end{aligned}
$$

By using (2.5) and (2.9), the estimate (2.27) takes the form

$$
\begin{aligned}
L^{\prime}(t) \geq & 2 \frac{\left[1-(3 / 4) \int_{0}^{t} g(s) d s\right]}{\left(1-\int_{0}^{t} g(s) d s\right)} H(t)+\left(\frac{\left[1-(3 / 4) \int_{0}^{t} g(s) d s\right]}{\left(1-\int_{0}^{t} g(s) d s\right)}-1\right)(g \diamond \nabla u)(t) \\
& +\left[1-\left(\frac{2}{p}+\frac{p-2}{p}\left(\frac{\alpha}{\beta}\right)^{p}\right) \frac{1-(3 / 4) \int_{0}^{t} g(s) d s}{\left(1-\int_{0}^{t} g(s) d s\right)}\right] \int_{\Omega}|u|^{p} d x \\
\geq & \gamma \int_{\Omega}|u|^{p} d x
\end{aligned}
$$

where

$$
\gamma=1-\left(\frac{2}{p}+\frac{p-2}{p}\left(\frac{\alpha}{\beta}\right)^{p}\right) \frac{1-(3 / 4) \int_{0}^{\infty} g(s) d s}{\left(1-\int_{0}^{\infty} g(s) d s\right)}>0
$$

because of (2.22). Next we have, by the embedding of the $L^{q}$ spaces,

$$
L^{p / 2}(t) \leq C\|u\|_{p}^{p}
$$


By combining (2.28) and (2.30) we get

$$
L^{\prime}(t) \geq \Gamma L^{p / 2}(t)
$$

A direct integration of (2.31) then yields

$$
L^{p / 2-1}(t) \geq \frac{1}{L^{1-p / 2}(0)-\Gamma t}
$$

Therefore $L$ blows up in a time $t^{*} \leq 1 / \Gamma L^{(p / 2)-1}(0)$.

\section{Acknowledgments}

The author would like to express his sincere thanks to King Fahd University of Petroleum and Minerals for its support. This work has been funded by KFUPM under project no. MS/VISCO ELASTIC/270.

\section{References}

[1] L. Alfonsi and F. Weissler, Blow up in $\mathbf{R}^{n}$ for a parabolic equation with a damping nonlinear gradient term, Nonlinear Diffusion Equations and Their Equilibrium States, 3 (Gregynog, 1989), Progr. Nonlinear Differential Equations Appl., vol. 7, Birkhäuser Boston, Massachusetts, 1992, pp. 1-20.

[2] J. Ball, Remarks on blow-up and nonexistence theorems for nonlinear evolution equations, Quart. J. Math. Oxford Ser. (2) 28 (1977), no. 112, 473-486.

[3] G. Da Prato and M. Iannelli, Existence and regularity for a class of integro-differential equations of parabolic type, J. Math. Anal. Appl. 112 (1985), no. 1, 36-55.

[4] A. Friedman, Mathematics in Industrial Problems. Part 5, The IMA Volumes in Mathematics and Its Applications, vol. 49, Springer, New York, 1992.

[5] V. K. Kalantarov and O. A. Ladyzhenskaya, The occurrence of collapse for quasilinear equations of parabolic and hyperbolic types, J. Sov. Math. 10 (1978), 53-70.

[6] H. A. Levine, Some nonexistence and instability theorems for solutions of formally parabolic equations of the form $P u_{t}=-A u+\mathscr{F}(u)$, Arch. Ration. Mech. Anal. 51 (1973), 371-386.

[7] H. A. Levine, S. Park, and J. Serrin, Global existence and nonexistence theorems for quasilinear evolution equations of formally parabolic type, J. Differential Equations 142 (1998), no. 1, 212-229.

[8] S. A. Messaoudi, A note on blow up of solutions of a quasilinear heat equation with vanishing initial energy, J. Math. Anal. Appl. 273 (2002), no. 1, 243-247.

[9] Blow up and global existence in a nonlinear viscoelastic wave equation, Math. Nachr. 260 (2003), 58-66.

[10] J. A. Nohel, Nonlinear Volterra equations for heat flow in materials with memory, Integral and Functional Differential Equations (Proc. Conf., West Virginia Univ., Morgantown, W. Va, 1979) (T. L. Herdman, H. W. Stech, and III S. M. Rankin, eds.), Lecture Notes in Pure and Appl. Math., vol. 67, Dekker, New York, 1981, pp. 3-82.

[11] P. Pucci and J. Serrin, Asymptotic stability for nonlinear parabolic systems, Energy Methods in Continuum Mechanics (Oviedo, 1994), Kluwer Academic Publishers, Dordrecht, 1996, pp. 66-74.

[12] H.-M. Yin, On parabolic Volterra equations in several space dimensions, SIAM J. Math. Anal. 22 (1991), no. 6, 1723-1737. 


\section{Blow-up of solutions of a heat equation}

[13] Weak and classical solutions of some nonlinear Volterra integrodifferential equations, Comm. Partial Differential Equations 17 (1992), no. 7-8, 1369-1385.

[14] J. N. Zhao, Existence and nonexistence of solutions for $u_{t}=\operatorname{div}\left(|\nabla u|^{p-2} \nabla u\right)+f(\nabla u, u, x, t)$, J. Math. Anal. Appl. 172 (1993), no. 1, 130-146.

Salim A. Messaoudi: Mathematical Sciences Department, King Fahd University of Petroleum \& Minerals, Dhahran 31261, Saudi Arabia

E-mail address: messaoud@kfupm.edu.sa 


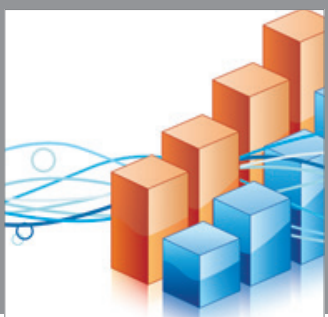

Advances in

Operations Research

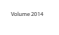

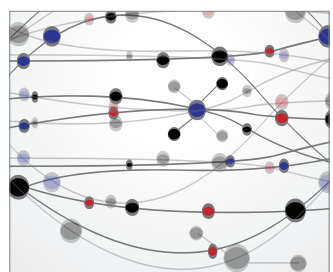

\section{The Scientific} World Journal
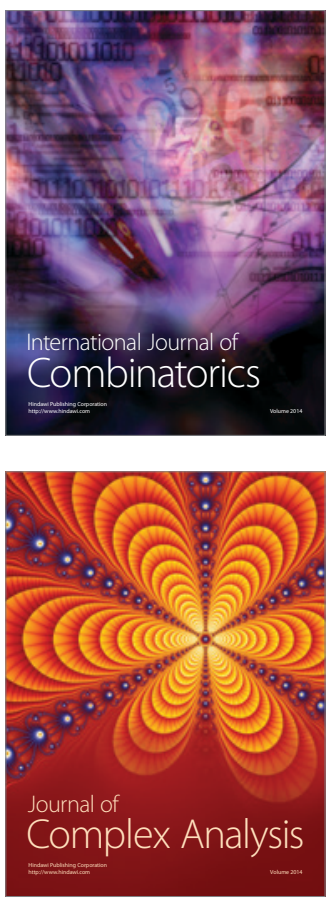

International Journal of

Mathematics and

Mathematical

Sciences
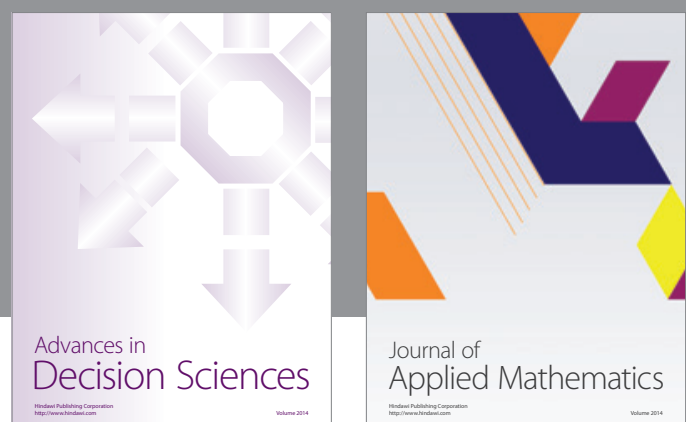

Journal of

Applied Mathematics
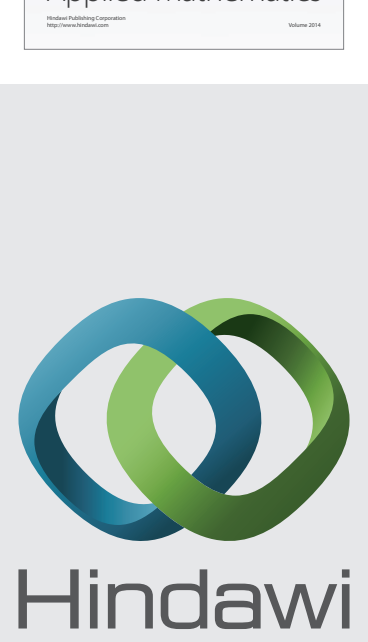

Submit your manuscripts at http://www.hindawi.com
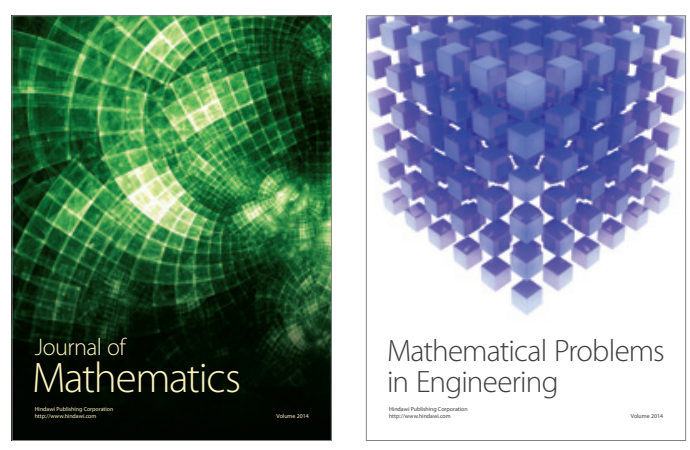

Mathematical Problems in Engineering
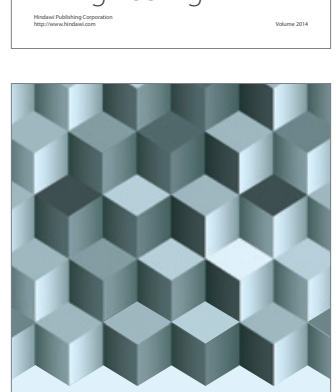

Journal of

Function Spaces
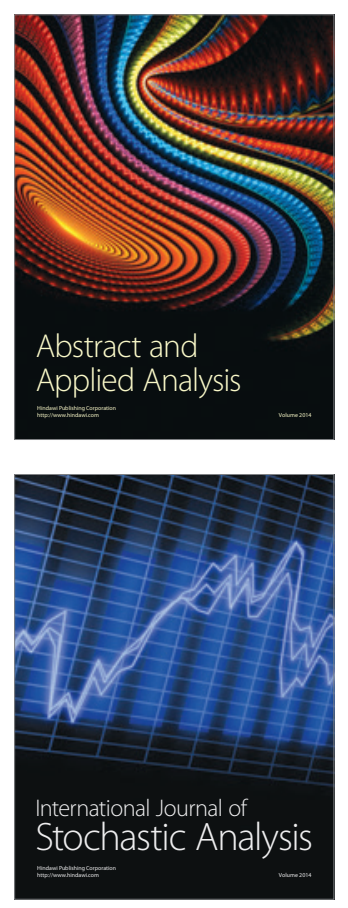

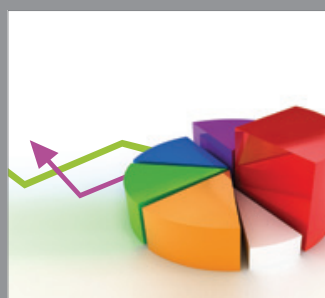

ournal of

Probability and Statistics

Promensencen
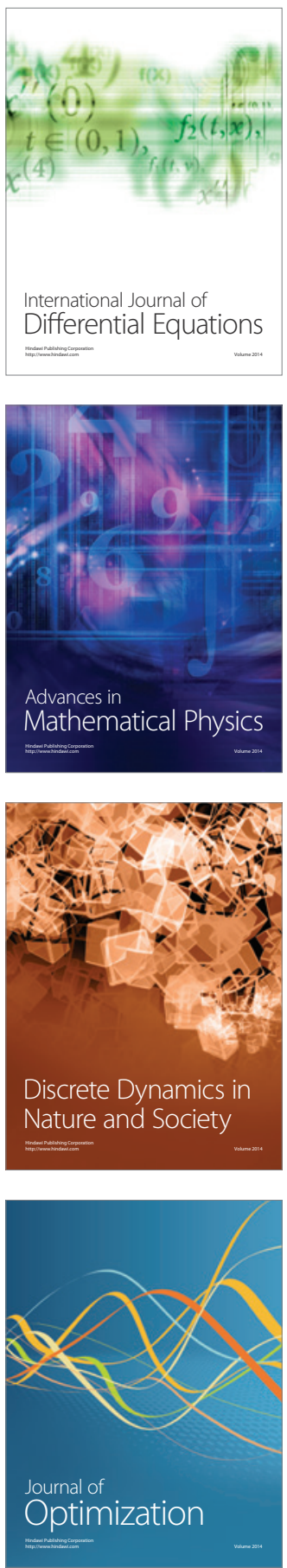